This PDF is a selection from a published volume from the National Bureau of Economic Research

Volume Title: Health Care Issues in the United States and Japan

Volume Author/Editor: David A. Wise and Naohiro Yashiro, editors

Volume Publisher: University of Chicago Press

Volume ISBN: 0-262-90292-7

Volume URL: http://www.nber.org/books/wise06-1

Conference Date: May 1-3, 2003

Publication Date: September 2006

Title: Geography and the Use of Effective Health Care in the United States

Author: Jonathan S. Skinner

URL: http://www.nber.org/chapters/c7365 


\title{
Geography and the Use of Effective Health Care in the United States
}

\author{
Jonathan Skinner
}

\subsection{Introduction}

There is a growing concern in the United States about shortfalls in health care quality. One influential Institute of Medicine (IOM) study called attention to problems of underuse, overuse, and misuse in health care quality (IOM 2000). With regard to underuse, there are a wide variety of procedures that are proven to be effective, yet are often used at rates as low as 50 percent for appropriate patients (most recently, see McGlynn et al. 2003). Examples of such effective treatments include the use of betablockers and aspirin for appropriate heart attack patients, annual eye examinations for people with diabetes, and mammography examinations for women over age fifty.

By the same token, there is evidence of overuse of procedures where they are not appropriate. For example, 20 percent of antibiotics prescribed in 1992 were used for common colds and respiratory tract infections, illnesses where the effectiveness of such antibiotics is questionable and may even be harmful. By the same token, only about one-third of angioplasties (PTCA) for cardiovascular disease are clearly appropriate, with about one-half uncertain and the remainder inappropriate (Schneider et al. 2001). Technological advances in diagnostic methods to detect appendicitis such as computerized tomography have improved tremendously, but there has been no apparent decline in the rate of inappropriate surgery for appendicitis (Flum et al. 2001). Finally, there is some evidence on the misuse of care, whether that means resulting complications resulting from errors in the

Jonathan Skinner is the John French Professor of Economics and professor of community and family medicine at Dartmouth College, and a research associate of the National Bureau of Economic Research. 
treatment of a disease or (more generally) whether it means a poor match between what the patient wants (in terms of risk and side effects) and what the patient actually receives (Wennberg, Fisher, and Skinner 2002).'

This paper focuses on the underuse of effective procedures and, in particular, the remarkable variation across regions in the United States with regard to the use of such treatments. Geographical variation in quality of care is of interest for two reasons. First, it provides a snapshot of the degree of technological inefficiency in the health care system; that is, how much do some regions and hospitals lag behind best-practice practiced in other areas? It is not surprising that health care innovations take time to diffuse; physicians need to be trained in the use of new technology (perhaps through residency programs), and their use spreads as the newly trained residents diffuse to new practice areas. What is more surprising is how persistent are the shortfalls in quality across regions.

For example, beta-blockers are used to reduce demands on weakened hearts following acute myocardial infarction (AMI), with well-established benefits in terms of reduced mortality. As Jencks, Huff, and Cuerdon (2003) reported, median state level of use among ideal patients is just 69 percent in 2000 and 2001. Yet beta-blockers have been known to be effective for many years; in 1985, Yusuf et al. concluded that "Long-term beta blockade for perhaps a year or so following discharge after an MI is now of proven value, and for many such patients mortality reductions of about $25 \%$ can be achieved" (335). In other words, despite the passage of nearly two decades since beta-blockers had become well established and inexpensive treatment for heart attacks, roughly 30 percent of AMI patients are still not getting appropriate treatment. ${ }^{2}$ Similarly, Garg et al. (2002) have found an average of 42 percent of heart attack patients for whom angiography was deemed "necessary" actually received the angiography, with regional rates varying from 24 to 58 percent.

To quantify the degree of technological process inefficiency in the United States, I use data from the Dartmouth Cardiovascular Atlas of Health Care (Wennberg and Birkmeyer 2000) that, in turn, is based on a large detailed survey (with chart reviews) of more than 160,000 heart attack patients during 1994 and 1995 . I find that the average loss per heart

I am grateful to helpful comments from seminar participants at the conference on health economics in Nikko, Japan in May 2003, and from Elliott Fisher, Douglas Staiger, and John E. Wennberg. This research was supported by a grant from the National Institute on Aging (PO1-AG).

1. Also see chapter 4 in the Economic Report of the President (Council of Economic Advisors 2002) for a parallel discussion of these issues.

2. Berwick (2003) makes this point with an analogy to the treatment of scurvy on sea-going ships; despite the fact that James Lancaster proved the effectiveness of vitamin C in the treatment of scurvy in 1601 (with randomization by treating just one ship in his fleet of four), it took more than 200 years before the British Navy enacted rules to ensure proper vitamin C consumption. 
attack patient, relative to best-practice care, is between $\$ 1,500$ and $\$ 5,000$ per year, depending on the benchmark used, the value of a life year, and other assumptions. The measured inefficiency does not stem from specific skills of the surgeon, but instead largely reflects the use (or nonuse) of pharmaceuticals such as beta-blockers and aspirin.

Geographic variation is used for a second task, to estimate a reduced form model of technology adoption that depends on supply factors that might be expected to lower the cost of adapting the new technology, such as the prevalence of cardiologists-who are presumably most aware of new technologies in the use of health care innovations-and demand factors such as education, income, and the overall incidence of heart disease in the region. Supply factors are less important in explaining technology diffusion than expected, but estimated demand effects are significant both statistically and economically. More cardiologists per capita is not significantly associated with higher rates of beta-blocker use, nor is there an association between cardiologists supply and the average quality use rate for beta-blockers, aspirin, reperfusion, and angiotensin converting enzyme (ACE) inhibitors.

In conclusion, there seems to be a missing link between the potentially large benefits of effective care for heart attack patients and financial incentives to pay for them. While beta-blockers may not be reimbursed directly by the Medicare program (and indeed cost just pennies per dose), other procedures with uncertain (or potentially negative) effects on outcomes, such as nonprimary angioplasty or angioplasty for non-Q-wave heart attacks, are paid in full by Medicare. ${ }^{3}$ An intriguing question that remains is why physicians working in hospital settings do not comply with quality guidelines, given the large benefits in terms of patient outcome to do so.

\subsection{Theoretical Models of Technology Adoption and Quality of Care}

Standard economic models begin with the assumption of profit maximization, and it is not surprising that such models predict that firms with the greatest expected gain from adopting new technology should be the ones to do so (e.g., Griliches 1957). In the context of health care, financial incentives are largely determined by the reimbursement policies of insurance programs, most notably the Medicare program. For most measures of effective care, however, the financial costs of adopting pharmaceutical technology is minimal, particularly for beta-blockers and aspirin where costs are measured in cents rather than in dollars.

The exception is reperfusion therapy for heart attack patients, which

3. Fisher and Skinner (2001) found a negative correlation between Medicare spending and Medicare quality. 
may consist either of primary angioplasty within twenty-four hours of the heart attack, or a regime of thrombolytic drugs, again administered shortly after the heart attack. The surgical approach, angioplasty, involves the use of a small deflated "balloon" inserted through a vein and threaded up to the blockage area, where it is popped open, thus generally restoring blood flow to the oxygen-starved heart muscle. The pharmaceutical approach uses "clot buster" thrombolytics that help to dissolve the platelets and other deposits that contribute to blocking blood flow to the heart; they are generally not used together because the thrombolytics discourages clotting, which can be dangerous during surgery. In this case, Medicare does compensate generously for surgery, thus providing a strong profit motive for investing in the newer technology. However, because the Medicare system is federal, it generally pays similar amounts across regions (albeit adjusted for costs of living, low-income patients, and graduate medical programs). While theoretical models would predict the more rapid diffusion of the most profitable (to hospitals or physicians) technologies, they do not generally predict why some regions would become persistently late adopters (see Skinner and Staiger 2005). Thus, one cannot appeal to differences in reimbursement rates to explain why regions in the United States differ so much with regard to technology adoption, particularly as the technologies I consider in the following are not particularly costly (i.e., aspirin or beta-blockers).

The literature in health care quality improvement is quite different and focuses much less on financial incentives and more on organizations and personalities. Indeed, the dominant model (adopted in turn from the management literature) sorts personalities of the relevant individuals according to whether they are innovators, early adopters, early or late majority adopters, and laggards (for an excellent review, see Berwick 2003). Some of these differences may, of course, stem from the organizational structure of the hospitals or the educational systems whence they emerge. As well, social networks (Coleman, Katz, and Menzel 1966) are another important mechanism for the transmission of medical innovations. These factors may be reflected in peer effects as found by Epstein and Nicholson (2005) and Burke, Fournier, and Prasad (2003).

We therefore consider quite general hypotheses based on a large class of factors on both the supply and demand side of health care markets. On the supply side, cardiologists might be expected to adopt earliest, as their practice is almost exclusively for patients with cardiovascular care, and they have received advanced training in cardiac care. As well, physicians would have the greatest incentive to adopt efficacious care the greater is the overall burden of cardiovascular disease or when urban dense regions increase the potential for specialization. On the other hand, demand-side factors could increase pressure on physicians to innovate; one might hypothesize that higher-educated or higher-income patients would be more familiar 
with new treatments and play a more active role in their use. (Conversely, they might be less likely to refuse the new treatment.)

This simple model suggests two distinct empirical exercises. The first is to attempt to quantify the gains from adopting more efficient technologies, which in the empirical section I define as the use of beta-blockers, ACE inhibitors, aspirin, and reperfusion in the treatment of AMI. Given those measures, factors (supply or demand) are next considered that may affect the adoption of one of these innovations in particular-both with regard to the use of beta-blockers and with regard to a set of effective treatments for AMI.

\subsection{Empirical Measures of Technology Adoption and Quality of Care}

In measuring technological diffusion for the treatment of heart attack patients, it is important to note that the technologies considered are not particularly hi-tech: beta-blockers and aspirin have been used for many years in the treatment of other illnesses, but their use for heart attack patients was established conclusively several decades ago (see Yusuf et al. [1985] and Heidenreich and McClellan [2001]). These treatments can be viewed as efficient care that is appropriate for nearly 100 percent of the appropriate patient population, regardless of health status or nearly any set of preferences. ${ }^{4}$ Problems with measuring health status, or with the true underlying preferences, are therefore greatly reduced, as everyone should demand the treatment, and the right rate does not depend on health status-as it is as close to 100 percent as one can get.

The disadvantage with measuring quality in this way is that it comprises a small fraction of how health care is actually practiced. The vast majority of decisions require much greater degrees of judgment, where a treatment choice may be appropriate and helpful for one patient but inappropriate and even harmful for another. For example, roughly 15 percent of angioplasty (a less invasive approach than bypass surgery to restoring blood flow to arteries in the heart) are deemed inappropriate, and about half are of uncertain benefit (Schneider et al. 2001). As well, treatment for chronically ill patients with diseases such as congestive heart failure, cancer, or pulmonary diseases differ widely across regions in the United States, and assessing how many physician visits are appropriate, or the appropriate level of intensive care unit (ICU) care, is difficult even with detailed clinical data. ${ }^{5}$

Some approaches focus on outcomes measures, such as mortality rates or complication rates, but such measures must first control for possible

4. The terminology here follows Wennberg, Fisher, and Skinner (2002).

5. This type of care, which accounts for nearly all of the variation across regions in the United States, is referred to as supply sensitive care because such care tends to be associated with the supply of hospital beds and the per capita supply of physicians. 
differences in the initial health status of the patient population. One example of an output measure of quality is the thirty-day or one-year mortality rate following AMI. The advantage of this type of measure is that it is a summary statistic and thus captures the multidimensional vector of inputs including the quality of the surgical team, follow-up care, smoking cessation programs, and the myriad other factors that comprise quality but which are difficult, if not impossible, to measure.

There are several shortcomings with measuring quality in this way, however. The first is simply the small numbers problem. If a hospital admits thirty AMI patients, purely random variation in the health status of the patient may lead to say six deaths in one year and nine deaths the next year. Thus, one cannot clearly determine whether the hospital looks better (or worse) than average because the hospital is better (or worse), or because the hospital had several lucky or unlucky draws. While McClellan and Staiger (1999) have used filtering methods at the hospital level to address this problem, it is still difficult to infer much about quality of care from smaller hospitals.

Another way around this problem is to aggregate individual hospitals into larger regions. This latter method was used in the Dartmouth Atlas of Health Care (Wennberg and Cooper 1999), creating 306 hospital referral regions (HRRs) based on the migration patterns of Medicare patients to hospitals (Wennberg and Cooper 1999). Each HRR is required to include at least one major hospital performing cardiovascular surgery and neurosurgery. While the larger size of the region masks the within-region (acrosshospital) variation in hospital quality, in practice there is plenty of acrossregion variation in these measures of output quality. However, it may be difficult to control entirely for differences across HRRs with respect to underlying health status, and so unexplained variations across regions may be due to unmeasured confounding variables rather than quality of care per se. More problematic is the improved detection of enzymes that are markers for AMI, and thus a patient who would be designated with unstable angina in one region could be labeled a non-Q-wave AMI in another region. To the extent these patients are healthier than average, the mortality estimates for that region could be biased downward. To avoid such biases, I adopt the process-based quality measures in this paper because at least for the treatment of AMI, they are highly predictive of outcomes.

\subsection{The Estimated Cost of Low-Quality Care for AMI}

An important study by Heidenreich and McClellan (2001) has attempted to quantify the incremental benefits of treatments for AMI that have proven effectiveness. They identify five treatments with "clear mortality benefit"-aspirin, beta-blockers, thrombolytics, ACE inhibitors, and primary angioplasty (i.e., done within twelve hours of the initial heart 
attack)-and provide consensus marginal odds ratios (i.e., their additional value conditional on other treatments) ${ }^{6}$ As well, they also provide odds ratios at thirty days of the marginal effectiveness of each procedure, conditional on the value of other treatments. The approach used here is to predict how mortality would vary across regions solely on the basis of the variations in the use of these effective procedures, multiplied by their effectiveness in expanding survival.

There are two adjustments to the data before deriving predicted mortality values in each region for the same hypothetical patient. The first is that the thirty-day odds ratios are assumed to be the same for one-year odds ratios. At least in the case of beta-blockers, this seems to be the case; the thirty-day odds ratio used in this study is 0.88 , while Krumholz et al. (1998) estimated a one-year risk ratio between 0.77 and $0.86 .^{7}$ The second adjustment is to combine angioplasty and thrombolytics as reperfusion. These procedures are typically substitutes (because thrombolytics increase the risk of bleeding during the angioplasty procedure), and just reperfusion rates are reported in the HRR-level data. I average their odds ratios to estimate effectiveness.

To estimate predicted mortality based solely on the HRR-level use of these four procedures, I further convert the odds ratios back into coefficients in the logistics regression and then calculate the following linear index:

$$
Z_{i}=\theta+\sum^{4} X_{i j} \gamma_{j}
$$

where $\gamma_{j}$ is the modified coefficient for the $j$ th treatment $(j=1,2,3,4), \theta$ the constant term, and $X_{i j}$ is the fraction of ideal candidates receiving treatment $j$ in HRR $i$. (Strictly speaking, one would like to know each individual and their treatment vector, but that data is not available; thus $I$ make a linear approximation in the context of the index.) Thus, $Z_{i}$ is the linear index of mortality in HRR $i$, and estimated mortality $m_{i}$ is $\exp \left(Z_{i}\right) /[1$ $\left.+\exp \left(Z_{i}\right)\right]$. The constant term $\theta$ is varied to ensure that the predicted mortality matches a 30 percent one-year mortality rate.

Data on use of these procedures is from the Cooperative Cardiovascular Project that collected chart review and outcome data on more than 160,000 heart attack patients during 1994 and 1995. The detailed information allowed researchers to define "ideal" and "appropriate" patients for each of the four treatments. Figure 8.1 shows the distribution of one of these treatments, beta-blocker use among ideal patients, for each of $267 \mathrm{HRRs}$. (The remaining HRRs were dropped because of small sample sizes.) There is a very large degree of variation across regions, with usage ranging from less than 20 percent to over 80 percent during 1994 and 1995.

6. They are aspirin $(0.77)$, beta-blockers $(0.88)$, thrombolytics $(0.75)$, primary angioplasty (0.51), and ACE inhibitors (0.94).

7. Note that odds ratios are typically larger in magnitude than risk ratios. 


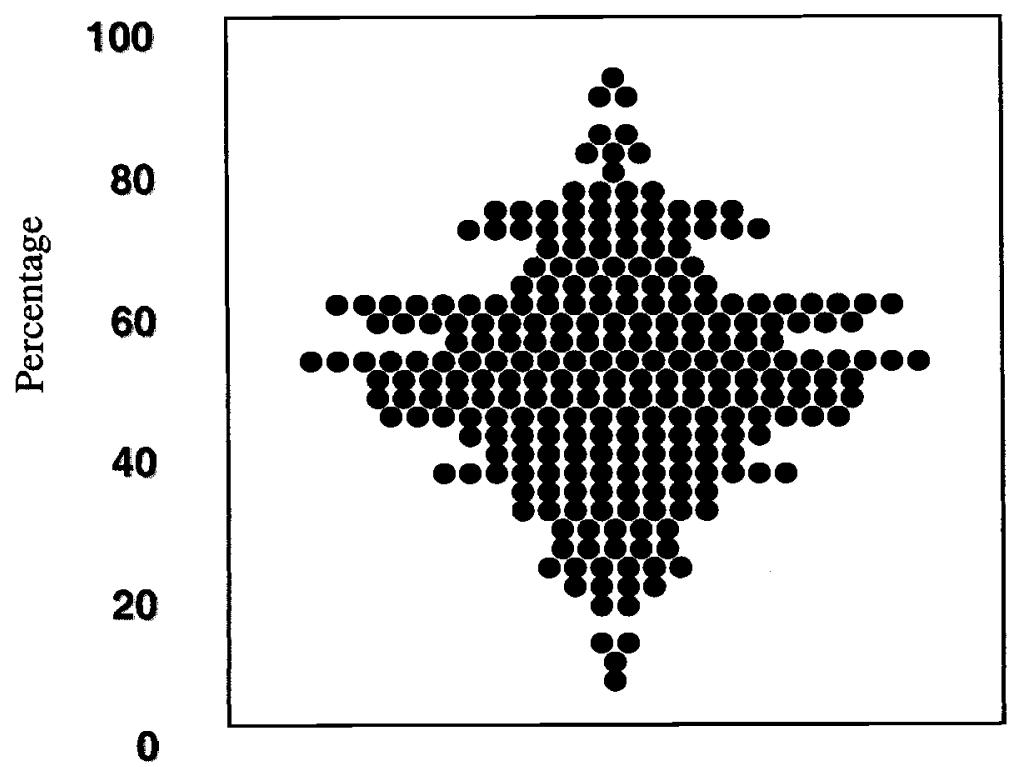

Fig. 8.1 Percent of "ideal" patients receiving beta-blockers at discharge following AMI (1994-1995)

Source: Dartmouth Atlas of Cardiovascular Health Care (Wennberg and Birkmeyer 2000). Note: Each dot refers to an HRR.

Predicted mortality, based on the variation in the utilization of these four treatments, ranges from less than 28 percent (in Everett, Washington [27.4 percent] and St. Petersburg, Florida [27.9 percent], for example) to more than 33 percent (Oxford, Mississippi [34.1 percent], Hattiesburg, Mississippi [33.3 percent] and Jonesboro, Arkansas [33.1 percent], for example). Using the adjustment from one-year mortality to expected life years used in Cutler et al. (1998) and placing $\$ 50,000$ as the price tag for the loss of one life year, the average efficiency cost per AMI patient is $\$ 3,687$ compared to the best performing region, Everett Washington. Compared to a uniform compliance rate of 90 percent across all four dimensions of quality, the average cost rises to $\$ 5,106$. The extremes, of course, are much greater; for patients living in Oxford, Mississippi who are appropriate candidates for these four treatments, they should be willing to pay $\$ 18,584$ to be airlifted to Everett, Washington.

These estimated values would be twice as high if one were to use a commonly used benchmark of $\$ 100,000$ per life year and even larger under some recommendations (Ubel, Hirth, Chernew, and Fendrick 2003). By the same token, however, one can deflate the values by the percentage of patients deemed "ideal" for the procedures. Taking a rough estimate of 39 percent of patients deemed ideal (as in Krumholz et al. [1998] for the case 
of beta-blockers) and applying this to all treatments yields a smaller mean cost of $\$ 1,446$ (compared to Everett, Washington) and $\$ 1,758$ (compared to a 90 percent uniform compliance rate) using the $\$ 50,000$ per life year price tag. A compromise estimate could be using these adjusted numbers with a $\$ 100,000$ per life year cost figure, leading to an estimate of approximately $\$ 3,000$ per person inefficient level, but with some regions showing benefits from better compliance as much as $\$ 10,000$ per patient. In other words, hospitals located in the poorly performing regions during 1994 and 1995 with 200 heart attack patients per year would have realized a social gain of more than \$2 million annually during 1994 and 1995 had they simply adopted best-practice cardiac care for heart attack patients. Any additional costs that would have been incurred would have been either negligible (aspirin, beta-blockers) or reimbursed by Medicare (angioplasty). Given the apparently large benefits associated with the adoption of this technology, I next turn to considering factors affecting its adoption or nonadoption.

\subsection{Factors Affecting the Adoption of High-Quality Care}

First consider supply factors that might affect the adoption of new technology. Figure 8.2 shows a graph of HRR-level beta-blocker use according

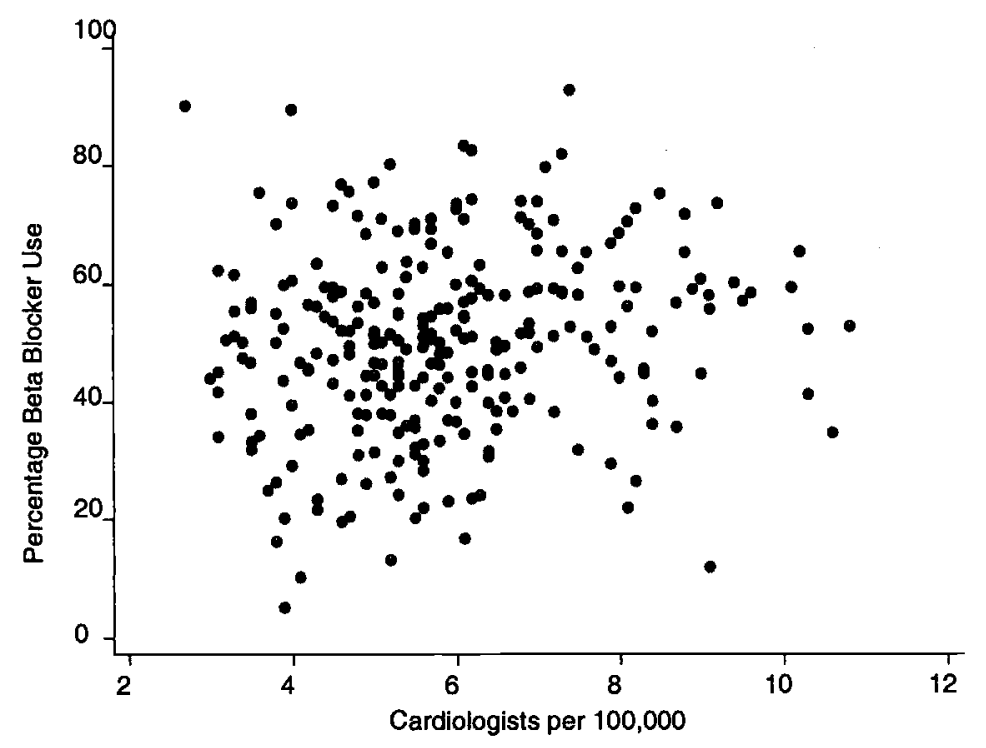

Fig. 8.2 Per capita cardiologist population and beta-blocker use

Source: Dartmouth Atlas of Cardiovascular Health Care (Wennberg and Birkmeyer 2000). Note: Each dot represents one of the 269 HRRs with sufficient information on beta-blocker use (that is, percentage of patients with AMI who are "ideal" for beta-blockers who get it). 
Table 8.1

Regression analysis explaining quality measures for cardiac care by HRR, 1994-1995

\begin{tabular}{|c|c|c|c|c|}
\hline & $\begin{array}{c}\text { Beta-blocker } \\
\text { use }^{a} \\
(1)\end{array}$ & $\begin{array}{l}\text { Aspirin } \\
\text { use in the } \\
\text { hospital } \\
\text { (2) }\end{array}$ & $\begin{array}{c}\text { Reperfusion } \\
\text { use }^{a} \\
(3)\end{array}$ & $\begin{array}{c}\text { Average } \\
\text { quality } \\
\text { measure } \\
\text { (4) }\end{array}$ \\
\hline Cardiologist per $100,000^{\mathrm{a}}$ & $\begin{array}{c}0.84 \\
(1.5)\end{array}$ & $\begin{array}{c}-0.31 \\
(1.8)\end{array}$ & $\begin{array}{c}-1.35 \\
(3.6)\end{array}$ & $\begin{array}{c}-0.20 \\
(0.8)\end{array}$ \\
\hline Percent college graduate ${ }^{b}$ & $\begin{array}{r}0.71 \\
(4.5)\end{array}$ & $\begin{array}{c}0.23 \\
(4.6)\end{array}$ & $\begin{array}{c}-0.02 \\
(0.2)\end{array}$ & $\begin{array}{r}0.37 \\
(5.4)\end{array}$ \\
\hline Poverty rate ${ }^{\mathrm{b}}$ & $\begin{array}{c}-0.82 \\
(5.2)\end{array}$ & $\begin{array}{c}-0.21 \\
(5.0)\end{array}$ & $\begin{array}{c}-0.25 \\
(2.4)\end{array}$ & $\begin{array}{c}-0.36 \\
(5.3)\end{array}$ \\
\hline Percent urban ${ }^{\mathrm{b}}$ & $\begin{array}{l}-0.16 \\
(2.7)\end{array}$ & $\begin{array}{l}-0.04 \\
(2.2)\end{array}$ & $\begin{array}{c}-0.02 \\
(0.5)\end{array}$ & $\begin{array}{c}-0.09 \\
(3.6)\end{array}$ \\
\hline Percent cigarette smokers ${ }^{\mathrm{c}}$ & $\begin{array}{l}-0.50 \\
(1.4)\end{array}$ & $\begin{array}{c}0.24 \\
(2.1)\end{array}$ & $\begin{array}{c}0.55 \\
(2.3)\end{array}$ & $\begin{array}{c}0.08 \\
(0.5)\end{array}$ \\
\hline AMI rate per $1,000^{d}$ & $\begin{array}{r}1.61 \\
(4.7)\end{array}$ & $\begin{array}{c}-0.06 \\
(0.6)\end{array}$ & $\begin{array}{c}-0.34 \\
(1.5)\end{array}$ & $\begin{array}{c}0.34 \\
(2.3)\end{array}$ \\
\hline Constant & $\begin{array}{l}29.82 \\
(2.4)\end{array}$ & $\begin{array}{r}83.27 \\
(20.9)\end{array}$ & $\begin{array}{l}74.52 \\
(8.9)\end{array}$ & $\begin{array}{r}61.10 \\
(11.0)\end{array}$ \\
\hline Adjusted $R^{2}$ & 0.30 & 0.16 & 0.10 & 0.26 \\
\hline Mean of dependent variable (SD) & $\begin{array}{c}50.89 \\
(13.71)\end{array}$ & $\begin{array}{l}86.12 \\
(4.29)\end{array}$ & $\begin{array}{l}67.00 \\
(8.37)\end{array}$ & $\begin{array}{l}66.04 \\
(5.61)\end{array}$ \\
\hline
\end{tabular}

Notes: $N=267$. Absolute value of $t$-statistics in parentheses. All regressions weighted by population of Medicare enrollees in 1996.

aSource: Wennberg and Birkmeyer (2000).

bSource: 1990 Census.

'Source: CDC Behavioral Risk Factor database.

¿Source: Wennberg and Cooper (1999).

to the supply of cardiologists per 100,000 population. There is a modest positive correlation; essentially having more cardiologists helps to ensure that the HRR is not in the "very low" category, with rates under 40 percent. Table 8.1 presents least squares regression results where the dependent variable is the percentage of ideal patients receiving a variety of highquality treatments as a function of a variety of factors, including an average measure of quality in the rightmost column.

The first column attempts to explain the use of beta-blocker use. The regression estimates imply that increasing the supply of cardiologists from the 10 th percentile $(4.2$ per 100,000$)$ to the 90 th percentile $(9.0$ per 100,000$)$ is predicted to increase the use of beta-blockers by just 4 percentage points, and even that estimate is not significantly different from zero at the 95 percent confidence interval. ${ }^{8}$ On the other hand, a greater burden of cardio-

8. By way of comparison, the standard deviation for beta-blocker use was 14 percentage points. 


\begin{tabular}{lc}
\hline City & $\begin{array}{c}\text { Percent of beta-blocker use } \\
\text { among ideal patients }\end{array}$ \\
\hline Albany & 90.0 \\
Rome & 68.7 \\
Columbus & 53.3 \\
Atlanta & $\mathbf{4 4 . 8}$ \\
Savannah & 35.8 \\
Macon & $\mathbf{2 1 . 3}$ \\
\hline
\end{tabular}

Source: Wennberg and Birkmeyer (2000).

Note: Medical schools are located in Macon and Atlanta (shown in bold).

vascular disease was associated with higher use of beta-blockers; a shift from the 10th to the 90th percentile in the underlying rate of AMI per 1,000 population of Medicare enrollees over age sixty-five was associated with a 5.6 percentage point increase in the use of beta-blockers. As well, demand factors appeared to exert an influence; shifting from the 10th to the 90th percentile in the percentage of college graduates living in the HRR was associated with a 5.8 percentage point higher rate of beta-blocker use.

Nor does the supply of cardiologists appear to be correlated with rates of aspirin use, reperfusion, or the combined index of cardiac health care quality (columns [2] through [4]). As was observed in the case of betablockers, most of the variation in the use of quality appeared associated with factors such as education levels and poverty rates in the region. Interestingly, the population of cardiologists was negatively associated with reperfusion rates. While the negative association holds solely for the per capita population of noninterventional cardiologists (that is, those who do not perform angioplasty), it is still somewhat surprising that reperfusion should not be more closely associated even with interventional cardiologists. This is a topic for future research.

To this point, the presence of a medical center has not yet been included in the statistical analysis. However, it may be of interest to consider a state chosen because of the very wide range in beta-blocker compliance, ranging from 21 percent in Macon, Georgia to 90 percent in Albany, Georgia. At least in Georgia, the use of beta-blockers in a community is not positively associated with the presence of a medical school; table 8.2 lists each HRR in Georgia, with the medical schools represented in bold. Indeed, Atlanta and Macon show beta-blocker use well below the state average.

\subsection{Conclusion}

The starting point for this analysis was the remarkable variation in measured quality of care for the treatment of heart attack patients across regions. 
This variation has also been observed in more recent studies across states (Jencks et al. 2000; Jencks, Huff, and Cuerdon 2003), with only a modest degree of convergence over time. The first question addressed in this paper is to quantify the extent of these inefficiencies across regions. The estimates are consistent with an average quality gap of between $\$ 1,800$ and $\$ 5,000$ per heart attack patient. These are estimates that are not compared to some hypothetical level of perfection, but instead relative to a region in the United States that has managed to make efficient use of the existing technological treatments for AMI. Nor do these estimates assume that delicate surgical skills can be effortlessly transferred across regions, as most of the gains are realized through pharmaceutical rather than surgical innovations.

We also ask what factors appear to be associated with the adoption of effective technology. The supply of cardiologists appears to be weakly associated with the use of beta-blockers, but not with an average of four quality measures. Instead, quality appears to be a normal good; regions with higher overall income and education levels are more likely to adopt early. As well, regions with a higher prevalence of cardiovascular disease are more likely to adopt beta-blockers and to experience higher average rates of effective care for AMI, suggesting that volume effects-the ability of hospitals to specialize in cardiac care where there are a relatively large number of patients with cardiac disease-may improve organizational environments for adopting newer technologies.

One unanswered question is why the medical profession is so slow to adopt newer technologies, despite the clear benefits of doing so. The traditional (noneconomic) diffusion model distinguishes between the "early adopters," who are most effective at disseminating the successful ideas of innovators to the four-fifths of the remaining population, and the "early majority" and "late majority" group, who, while not entirely laggards, account for the majority of the profession in being very slow to adopt new innovations. For example, Berwick (2003) suggests that

Medical communities are primarily local in their orientation, are dominated numerically by early and late majority groups, and do not trust remote and personally unfamiliar sources of authority. (1973)

A topic for future research is therefore to identify whether regions that are more effective in adopting new technologies have a "flatter" structure of authority as opposed to a hierarchical one where a "late majority" department chair can implicitly hold up new innovations for decades.

Regions that score well on treatment for AMI are only modestly more likely to score well for other measures of process quality. The correlation coefficient between beta-blocker use and the provision of annual eye examinations for diabetics in the Medicare population is $0.15(p<.02)$, while the corresponding coefficient with mammography rates is $0.29(p<.001)$. The low correlation across regions, as well as the low explanatory value of 
the regressions explaining adoption, are consistent with a model where each physician group, whether cardiologists, cardiac surgeons, internists, or family practice physicians, undergo different processes regarding the adoption of specific technologies for their group.

This is not to say, however, that financial incentives introduced with regard to these specific quality measures may not exert a strong and important impact on their overall use. One of the reasons why the use of betablockers grew so rapidly between 1997 and 1998 and 2000 and 2001 is because it was one of the Health Plan Employer Data and Information Set (HEDIS) quality measures used to quantify health care quality at the hospital or provider level. That there is so much latitude in the potential to improve the quality of care (and at such little cost) suggests that the incentives of the Medicare program could be better aligned with improving quality rather than rewarding the quantity of services in the United States.

\section{References}

Berwick, D. M. Dissemination innovations in health care. 2003. Journal of the American Medical Association 289 (15): 1969-75.

Burke, M., G. M. Fournier, and K. Prasad. 2003. Physician social networks and geographical variation in medical care. The Center on Social and Economic Dynamics Working Paper no. 33. Washington, DC: Brookings Institution.

Coleman, J. S., E. Katz, and H. Menzel. 1966. Medical innovation: A diffusion study. Indianapolis, IN: Bobbs-Merrill.

Council of Economic Advisors. 2002. Economic report of the president. Washington, DC: Government Printing Office.

Epstein, A., and S. Nicholson. 2005. The formation and evolution of physician treatment styles: An application to cesarean sections. NBER Working Paper no. 11549. Cambridge, MA: National Bureau of Economic Research, August.

Fisher, E., and J. Skinner. 2001. Medicare reform: Don't spend more, spend right. Providence Journal (March).

Garg, P. P., M. B. Landrum, S.-L. Normand, J. Z. Ayanian, P. J. Hauptman, T. J. Ryan, B. J. McNeil, and E. Guadagnoli. 2002. Understanding individual and small area variation in the underuse of coronary angiography following acute myocardial infarction. Medical Care 40 (7): 614-26.

Griliches, Z. 1957. Hybrid corn: An exploration in the economics of technological change. Econometrica 25 (4): 501-22.

Heidenreich, P. A., and M. McClellan. 2001. Trends in treatment and outcomes for acute myocardial infarction 1975-1995. The American Journal of Medicine 110: $165-74$.

Institute of Medicine (IOM). 2000. To err is human: Building a safer health system, ed. Linda T. Kohn, Janet M. Corrigan, and Molla S. Donaldson. Washington, DC: National Academy Press.

Krumholz, H. M., M. J. Radford, Y. Wang, J. Chen, A. Heiat, and T. A. Marciniak. 1998. National use and effectiveness of $\beta$-blockers for the treatment of elderly patients after acute myocardial infarction. JAMA 280 (7): 623-29. 
Jencks, S. F., T. Cuerdon, D. R. Burwen, B. Fleming, P. M. Houck, A. E. Kussmaul, D. S. Nilasena, D. L. Ordin, and D. R. Arday. 2000. Quality of medical care delivered to Medicare beneficiaries: A profile at state and national levels. JAMA 284 (13): 1670-76.

Jencks, S. F., E. D. Huff, and T. Cuerdon. 2003. Change in the quality of care delivered to Medicare beneficiaries. $J A M A 289$ (3): 305-12.

McClellan, M. B., and H. Noguchi. 1998. Technological change in heart-disease treatment: Does high-tech mean low value? American Economic Review 88 (2): 90-96.

McClellan, M., and D. Staiger. 1999. The quality of health care providers. NBER Working Paper no. 7327. Cambridge, MA: National Bureau of Economic Research, August.

McGlynn, E. A., S. M. Asch, J. Adams, J. Keesey, J. Hicks, A. DeCristofaro, and E. A. Kerr. 2003. The quality of health care delivered to adults in the United States. New England Journal of Medicine 348 (26): 2635-45.

Schneider, E. C., L. L. Leape, J. S. Weissman, R. N. Piana, C. Gatsonis, and A. M. Epstein. Racial differences in cardiac revascularization rates: Does "overuse" explain higher rates among White patients? Annals of Internal Medicine 135 (5): $328-37$.

Skinner, J., and D. Staiger. 2005. Technological Diffusion from hybrid com to betablockers. NBER Working Paper no. 11251. Cambridge, MA: National Bureau of Economic Research, March.

Ubel, P. A., R. A. Hirth, M. E. Chernew, and A. M. Fendrick. 2003. What is the price of life and why doesn't it increase at the rate of inflation? Archives of Internal Medicine 163 (14): 1637--41.

Wennberg, D. E., and J. Birkmeyer, eds. 2000. The Dartmouth atlas of cardiovascular health care. Chicago: American Hospital Association.

Wennberg, J. E., and M. Cooper, eds. 1999. Dartmouth atlas of health care 1999. Chicago: Dartmouth Medical School and American Hospital Association.

Wennberg, J. E., E. Fisher, and J. Skinner. 2002. Geography and the debate over Medicare reform. Health Affairs (Web Exclusive): W96-W114.

Yusuf, S., R. Peto, J. Lewis, R. Collins, and P. Sleight. 1985. Beta blockage during and after myocardial infarction: An overview of the randomized trials. Progress in Cardiovascular Disease 27 (5): 335-71. 